\title{
Simulation of electrooptical measurements of prebreakdown electric fields in water. Part 2. Electric field near cathode streamer
}

This paper was downloaded from TechRxiv (https://www.techrxiv.org).

\section{LICENSE}

CC BY-NC-SA 4.0

SUBMISSION DATE / POSTED DATE

26-02-2022 / 03-03-2022

\section{CITATION}

Yassinskiy, Vladimir; Kuznetsova, Yulia; Korobeynikov, Sergey; Vagin, Denis (2022): Simulation of electrooptical measurements of prebreakdown electric fields in water. Part 2. Electric field near cathode streamer. TechRxiv. Preprint. https://doi.org/10.36227/techrxiv.19242687.v1

$\mathrm{DOI}$ 


\title{
Simulation of electrooptical measurements of prebreakdown electric fields in water. Part 2. Electric field near cathode streamer
}

\author{
V.B. Yassinskiy ${ }^{1}$, Yu.A. Kuznetsova ${ }^{1}$, S.M. Korobeynikov ${ }^{2}$, and D.V. Vagin ${ }^{2}$ \\ ${ }^{1}$ Karaganda Technical University, Kazakhstan. \\ ${ }^{2}$ Novosibirsk State Technical University, Russia
}

\begin{abstract}
In this paper, we continue the analysis of electrooptical studies of the prebreakdown stage in water in the submicrosecond range. We consider a cathode streamer developing in a system of the spherical electrodes. Computer simulation of electric showed that at a constant $\varepsilon=80$, the field strength at the head of the cathode streamer should not exceed $E=1.9 \mathrm{MV} / \mathrm{cm}$. Taking into account the nonlinearity of the permittivity $\varepsilon=\varepsilon(E)$ made it possible to estimate this field strength as $E \sim 2.4-3.1 \mathrm{MV} / \mathrm{cm}$. It is shown that even with some excess of the strength over the threshold value $\left(E_{S} \sim 3 \mathrm{MV} / \mathrm{cm}\right)$, at which the state of dipole saturation is possible; its consideration does not lead to a noticeable change in the result. Thus, a significant decrease in the field strength near the cathode streamer compared to the anode streamer was confirmed.
\end{abstract}

Index Terms - electric field measurements, cathode streamer, Kerr effect, mathematical simulation.

\section{INTRODUCTION}

Plasma processes in water have many applications - water purification, decontamination, biomedicine, nanosciences [1-3], development of high-voltage switches, generation of pressure pulses, creation of pulsed capacitive energy storage devices [4-7], and so on.

Depending on the conditions for the initiation of a discharge during the liquid breakdown, it can begin both from the anode and from the cathode. Usually, a streamer is a harbinger of the appearance of a breakdown channel. The papers [2, 4, and 5] provide reviews of the conditions for the initiation of such processes. Consideration of the electric field of the streamer in the process of the origin of the plasma region and in the process of its development is the subject of the works of several groups of researchers [58]. Estimates show that in the case of a discharge from the cathode, the streamer field strength is considered to be significantly lower than that of the anode streamer. But these estimates are mostly indirect and vary greatly among different researchers.

In each case, the key question remains unclear: what field strength is necessary for the appearance of an anode or cathode streamer?

The difference in the magnitudes of the estimates of the value of the field is enormous. Thus, in [9], values of about $1 \mathrm{MV} / \mathrm{cm}$ are indicated, in [10] $-1.7-2.2 \mathrm{MV} / \mathrm{cm}$. in [11] for the cathode streamer $E \sim 2$ $\mathrm{MV} / \mathrm{cm}$, in [3] $E \sim 1-1.8 \mathrm{MV} / \mathrm{cm}$. The review article [5] considers the mechanisms of ignition and advancement of a streamer at $E \sim 1-10 \mathrm{MV} / \mathrm{cm}$, in [12] $E \sim 20-30 \mathrm{MV} / \mathrm{cm}$. The highest values $\sim 220 \mathrm{MV} / \mathrm{cm}$ were obtained in [13] for a subnanosecond breakdown during streamer advancement. Part 1 of this paper [14] concerns the electric field $E$ estimates near point anode to the moment of streamer initiation. It was found extremely high values of $E \sim 40-50 \mathrm{MV} / \mathrm{cm}$. An increase in the field strength at the ionization wavefront by $50 \%$ of the applied voltage up to $1.6 \mathrm{MV} / \mathrm{cm}$ was recorded in [15]. The appearance of a cathode streamer at an average intensity of $\sim 300 \mathrm{kV} / \mathrm{cm}$ was recorded in [16].

The purpose of our work is to estimate the field strength near the boundary of the cathode streamer 
using the kerrogram obtained in [11] based on the modeling technique proposed by us [17].

\section{ANALYSIS AND MODELING OF THE EXPERIMENTAL KERROGRAM}

The results of electrooptic measurements described in [16] were selected for analysis.

The experiments were carried out by the authors of [16] in a measuring cell (see Fig. 1) with two spherical stainless steel electrodes with a radius of $50 \mathrm{~mm}$ and a gap of $4 \mathrm{~mm}$.

The cell was filled with deionized water with a specific conductivity $\sigma \approx 10^{-7}(\mathrm{Ohm} \cdot \mathrm{cm})^{-1}$ coming from a closed purification loop. A voltage was applied to the electrodes with a characteristic rise time $\tau_{f} \approx 0.6 \mu$ s and amplitude of $160 \mathrm{kV}$.

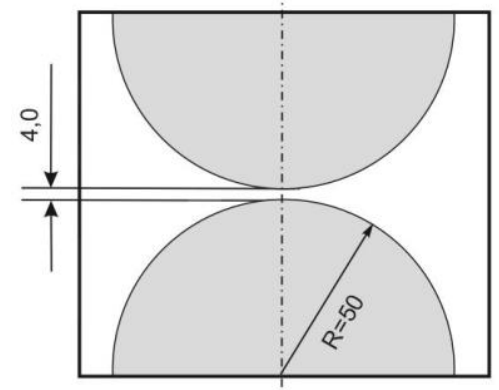

Fig. 1. Spherical electrode system.

On fig. 2 it shows the original kerrogram taken with initially crossed polarizers. The almost uniform illumination of the registration field indicates that nowhere in the discharge gap does the phase incursion become less than $\pi$. A dark Kerr fringe is seen above the streamer propagating from the cathode. This is possible if the field is distorted by a growing streamer. This is the distortion we tried to estimate using computer simulation according to the method described in [17].

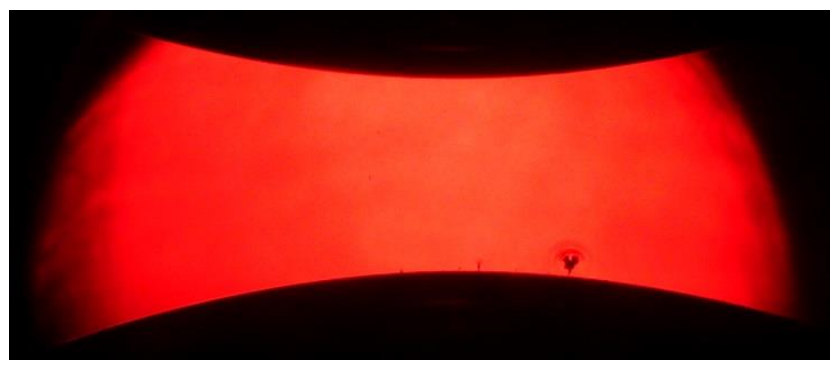

a)

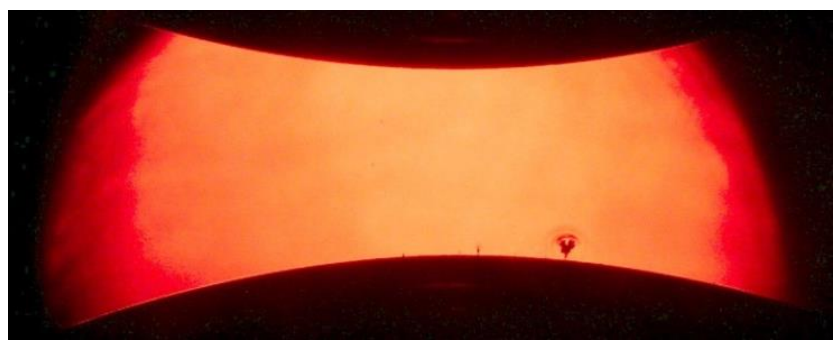

b)

Fig. 2. Original kerrogram (a), after increasing the contrast $(b)$

For the convenience of analysis, the original kerrogram image (Fig. 2a) was processed in a graphical editor (Fig. 2b), which made it possible to more clearly distinguish changes in the near streamer zone. With a gap between the electrodes of $4 \mathrm{~mm}$, the streamer is at a distance of $2.4 \mathrm{~mm}$ from the symmetry axis of the measuring cell. Checking the magnitude and configuration of the electric field near the cathode showed (see Fig. 3) that the conditions on the symmetry axis and at the place where the streamer appeared are almost identical. Therefore, an axisymmetric problem was solved, with the location of the streamer on the axis of symmetry of the measuring cell. 


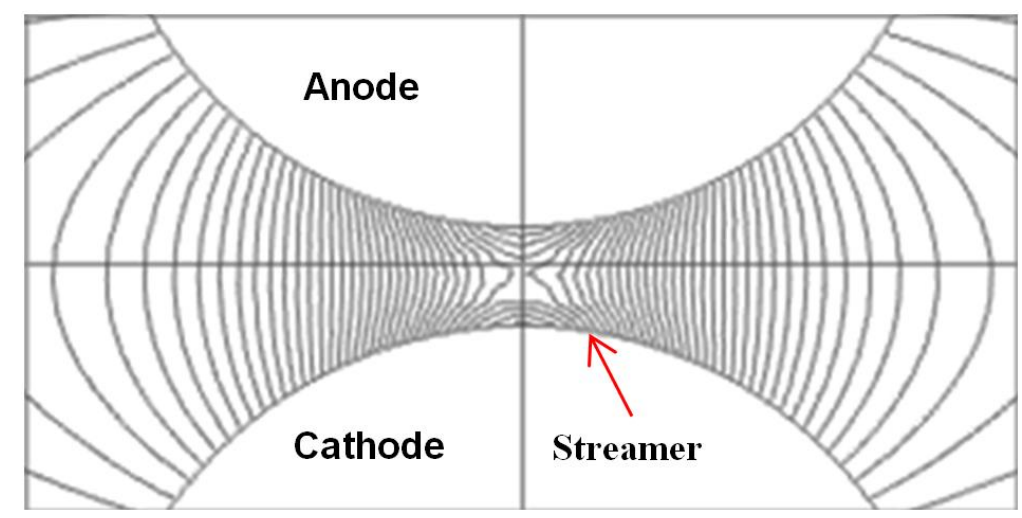

Fig. 3. Isolines of the electric field intensity

For analysis, a 3D model of the measuring cell was created (Fig. 1), a fragment of the kerrogram with a streamer was enlarged (see Fig. 4a), and a scaled 3D model of the streamer was placed on the cathode. At the first stage, the streamer model was simplified (see Fig. 4b).

The problem was solved by the finite element method. In this case, it was assumed that the electrical conductivity of the streamer body is high enough to displace the field from the streamer region. In addition to the geometric characteristics, the computer simulation fully reproduced all the optical and electrical parameters of the experiment.

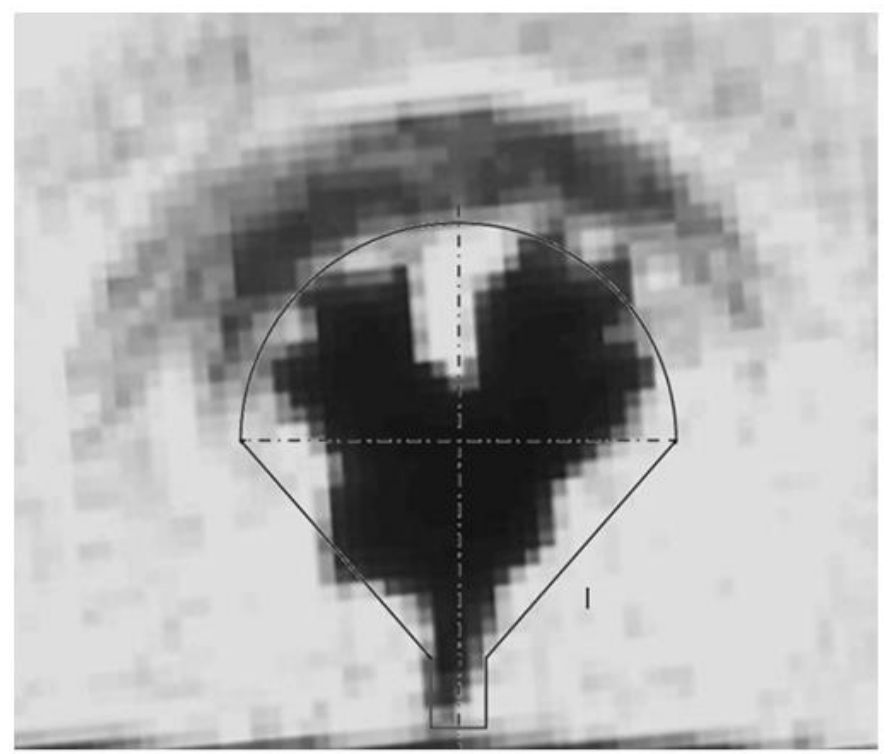

a)

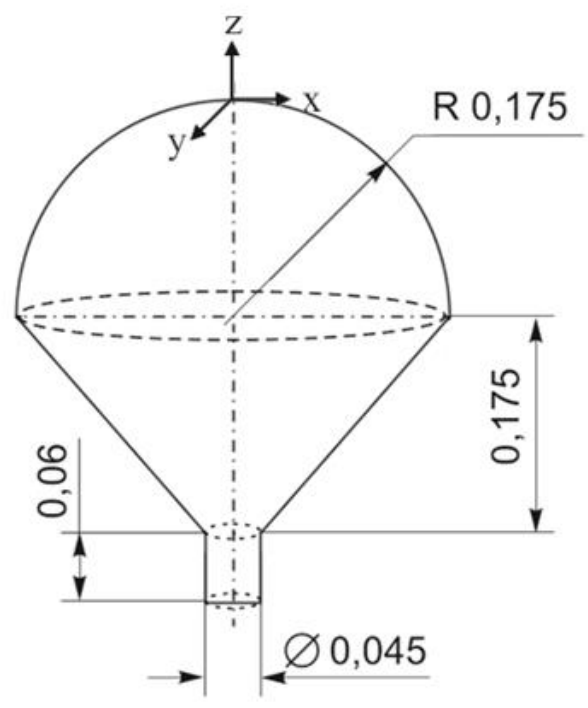

b)

Fig. 4. (a) is an enlarged fragment of the kerrogram from Fig. 2b, $(b)$ is streamer model

The area above the upper point of the streamer was considered. The height of the streamer model is $410 \mu \mathrm{m}$. The dimensions of the computational domain are $\mathrm{z} \times \mathrm{X}=200 \times 400 \mu \mathrm{m}$. The origin of coordinates of the region under consideration was located at a point on the upper surface of the head of the streamer model. The discretization step of calculations is $2.5 \mu \mathrm{m}$.

If necessary, the settlement zone was adjusted in certain areas. 


\section{DISCUSSION}

The kerrogram shown in Fig. 2 was obtained by setting the Kerr cell to maximum transmission, that is, the polarizers were installed perpendicular to each other at an angle of $45^{\circ}$ to the field direction. In this case, the relative intensity of the transmitted light is given by:

$$
I(x, z)=I_{0} \sin ^{2} \frac{\Delta \Phi_{Y}(x, z)}{2} .
$$

Here $\Delta \Phi_{Y}$ is the phase difference between the ordinary and extraordinary rays on the way across the measuring cell along the $\mathrm{Y}$-axis.

With the named set of initial data, calculations were performed in the near-electrode zone with a streamer model (see Fig. 4b) according to the method [17].

On fig. 5 shows the visualization of the calculations.

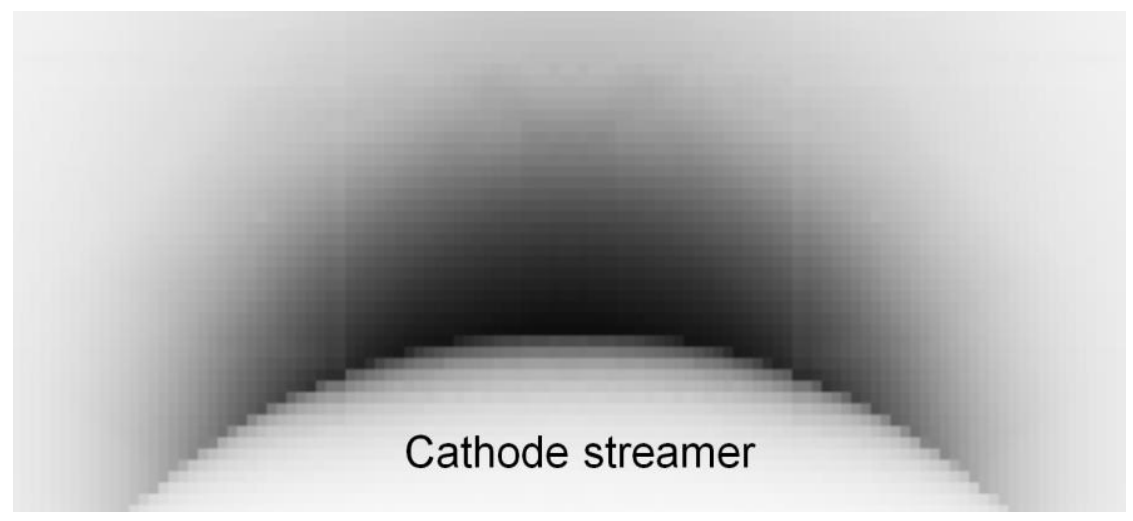

Fig. 5. Visualization of the calculated kerrogram

For greater clarity, Fig. 6 shows a scale comparison of the resulting picture with a real kerrogram.

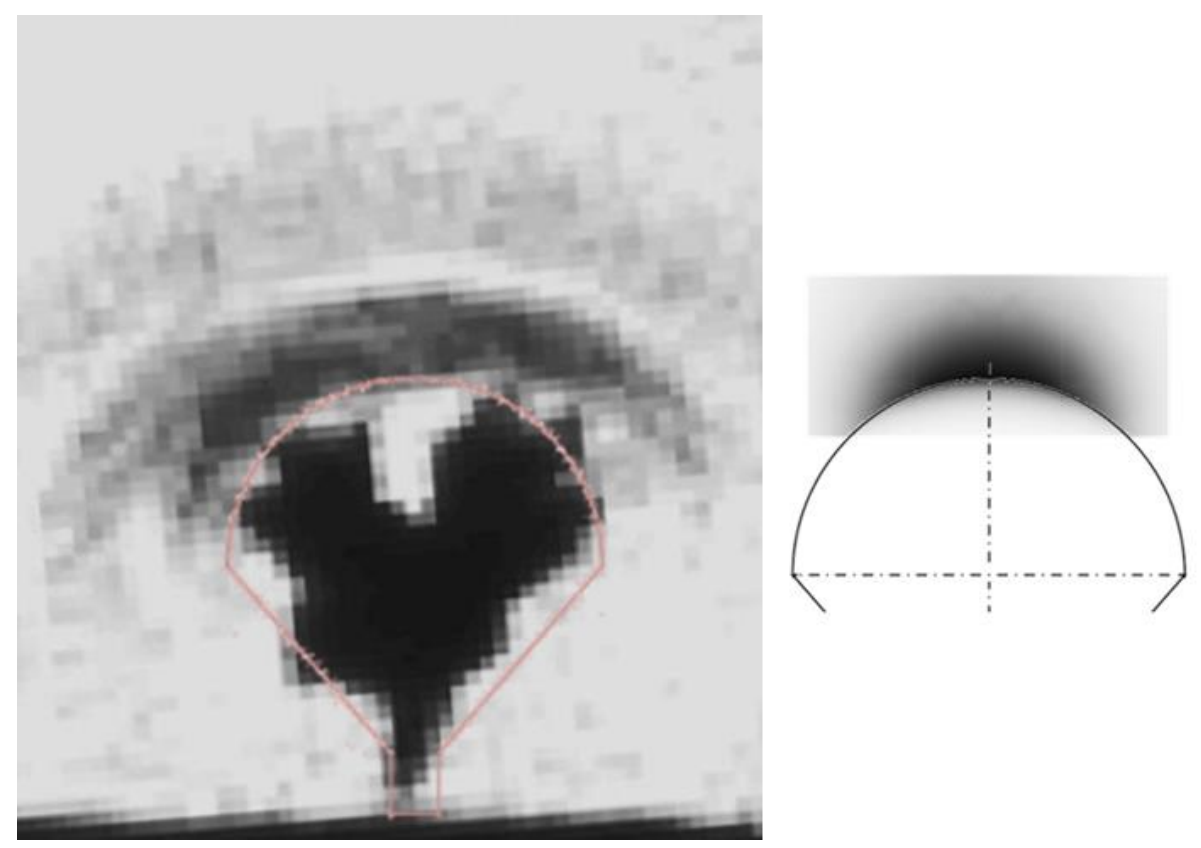

Fig. 6. Comparison of real and calculated kerrograms. The contours of the model are superimposed on the experimental kerrogram. 
It can be seen that, qualitatively, the calculated kerrogram in the region of the symmetry axis is very similar to the experimental one. The height of the computational zone above the head of the streamer model was $70 \mu \mathrm{m}$ with a gap between the electrodes of $4 \mathrm{~mm}$. Therefore, it was viewed (Fig. 7) how the relative intensity $\mathrm{I} / \mathrm{I}_{0}$ and the phase shift $\Delta \Phi / \pi$ change over the entire discharge gap.

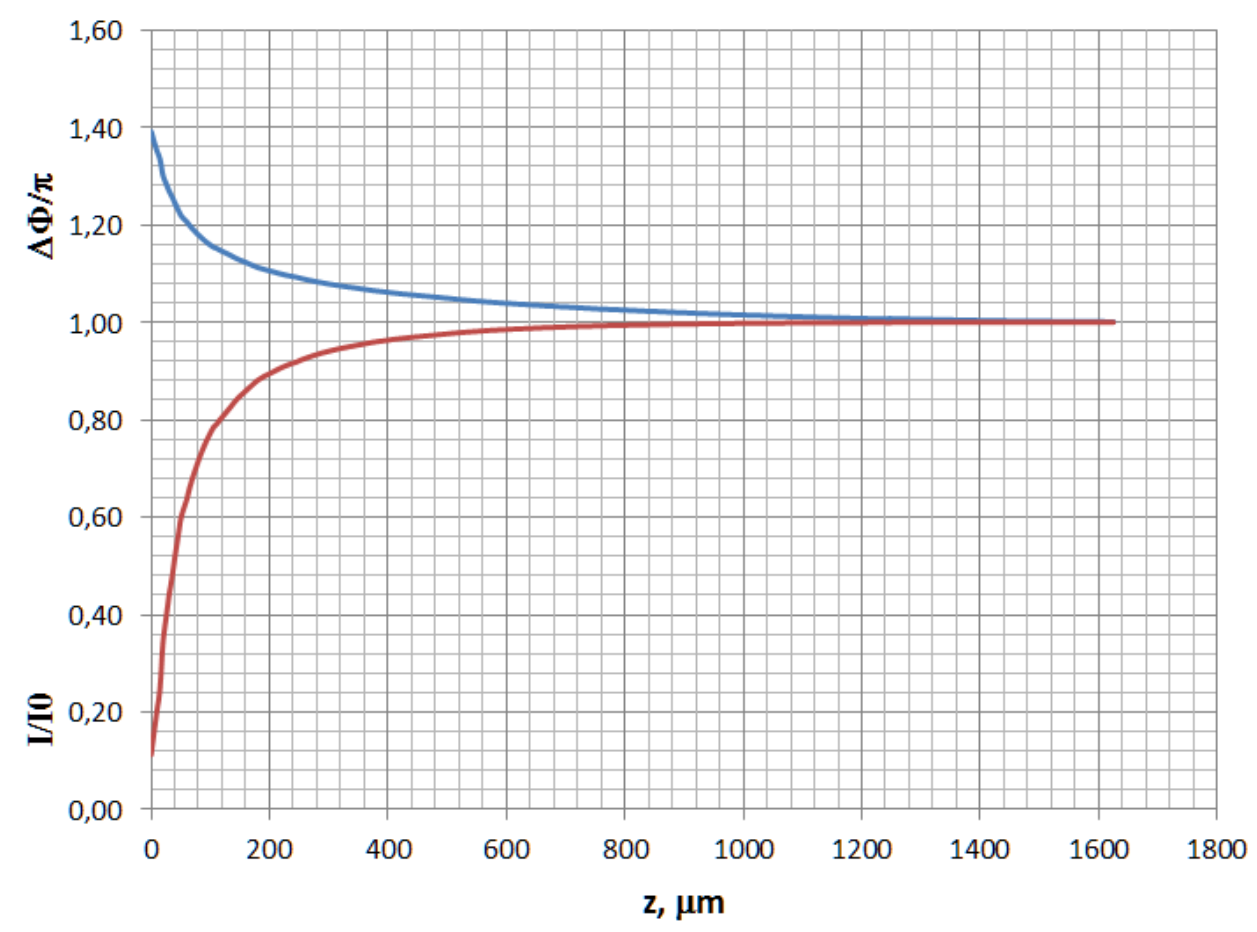

Fig. 7. Relative intensity $\mathrm{I} / \mathrm{I}_{0}$ and phase shift $\Delta \Phi / \pi$ along the z-axis.

The phase difference $\Delta \Phi / \pi$ was determined within the mathematical model by the formula:

$$
\frac{\Delta \Phi(x, z)}{\pi}=2 B \cdot \int_{\ell_{1}}^{\ell_{2}} E_{\perp}^{2}(y) d y .
$$

Here $B$ is the Kerr constant, $E_{\perp}$ is the projection of the electric field strength vector onto the $\mathrm{z}$ axis. The validity of replacing $E$ with $E_{\perp}$ was proved in [17].

It can be seen from the graph that the visibly dark band almost ends already at $\mathrm{z}=70 \mu \mathrm{m}$ - there $\mathrm{I} / \mathrm{I}_{0}=$ 0.68. Farther from the streamer, the field of view continues to brighten and, starting from $0.885 \mathrm{~mm}, \mathrm{I} / \mathrm{I}_{0}$ and $\Delta \Phi / \pi$ are exactly 1 , that is, the entire discharge gap becomes completely bright. This value fully correlates with the real kerrogram and corresponds to expression (1). (See Fig. 2, 4, 6, 7).

Calculations of the field strength distribution were carried out under two different initial conditions. The first condition is the neglect of the possible nonlinearity of the polarization. In this case, the calculations were carried out at a constant relative permittivity $\varepsilon=80$. Then, since $\varepsilon$ still depends nonlinearly (Fig. 8 ) on the field strength [18], the same calculations were carried out for these conditions. 


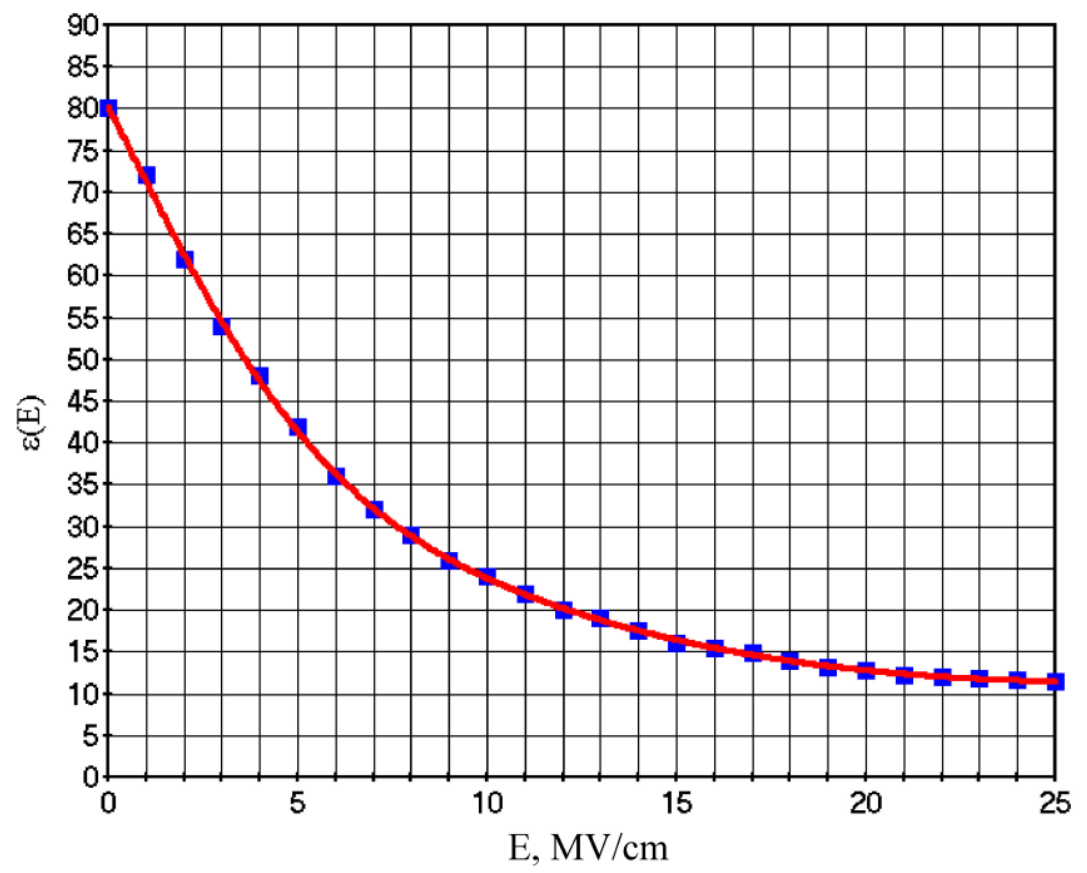

Fig. 8. Dependence of $\varepsilon$ on $E$ [18]

Nonlinearity $\varepsilon$ was taken into account as follows.

The distribution of a stationary electric field in a non-conducting medium is described by the equation

with boundary conditions

$$
-\operatorname{div}(\varepsilon \cdot \operatorname{grad} V)=0
$$

$$
\left.V\right|_{S_{1}}=0,\left.V\right|_{S_{2}}=U,\left.\frac{d V}{d n}\right|_{S_{3}}=0 .
$$

Here $\varepsilon=\varepsilon(E)$ is the relative permittivity of the substance. $V$ is the scalar potential of the electric field such that $\boldsymbol{E}=-\operatorname{grad} V, \boldsymbol{E}$ is the vector of the electric field strength, $\mathrm{S}_{1}$ and $\mathrm{S}_{2}$ are the surfaces of the electrodes, $U$ is the potential difference between the electrodes, and $S_{3}$ is the remote boundary of the computational domain. Since $\varepsilon$ depends nonlinearly on $E$ [18], then equation (2) is also nonlinear.

As a result, the electric field strength distribution was obtained at different distances from the streamer.

Comparison of calculations with constant $\varepsilon$ and $\varepsilon=\varepsilon(E)$ showed that the values of the electric field strength at the leading edge of the cathode streamer with a simplified model lie within 1.9-2.2 MV/cm (Fig. 9). 


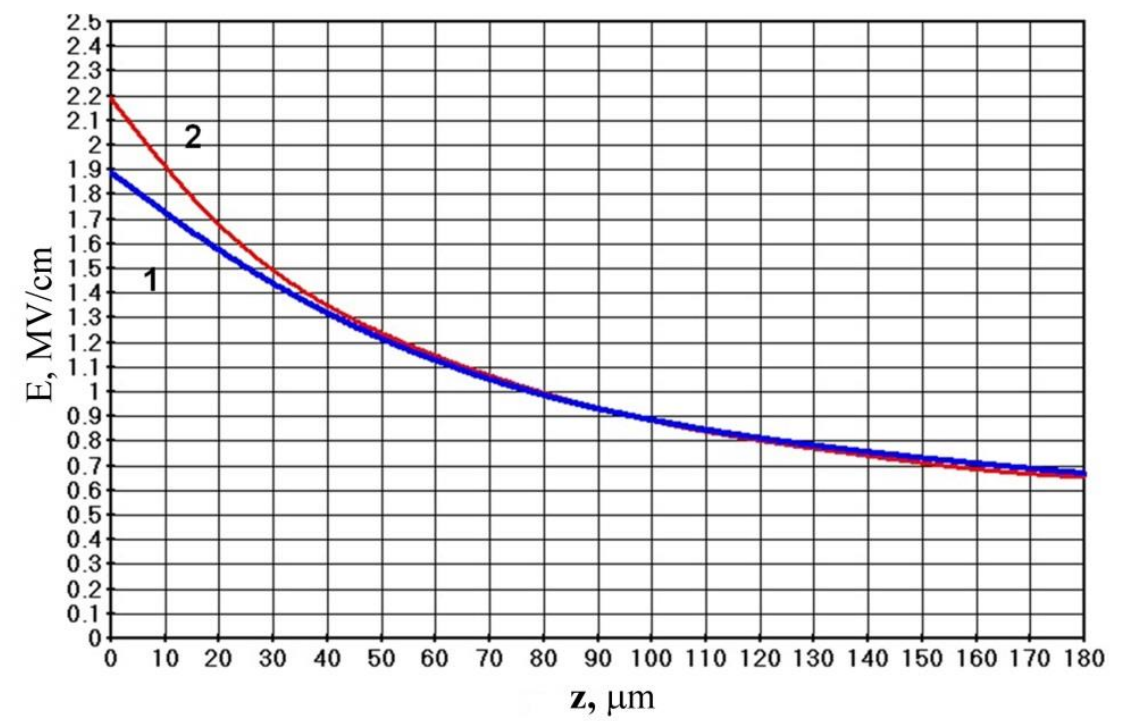

Fig. 9. The electric field strength distribution at different distances from the streamer: 1 is $\varepsilon=80,2$ is

$$
\varepsilon=\varepsilon(\mathrm{E})
$$

That is, taking into account the nonlinearity $\varepsilon$ leads to an increase in electric field by $13 \%$. In our case, the field strength $\mathrm{E}=2.2 \mathrm{MV} / \mathrm{cm}$ does not reach the threshold value $E \sim 3 \mathrm{MV} / \mathrm{cm}$ even when taking into account the nonlinearity $\varepsilon$. That is, in contrast to the anode streamer, dipole saturation does not occur within the considered streamer model.

The mathematical model used by us made it possible to find out the distribution of the phase shift $\Delta \Phi / \pi$ with distance from the streamer head (Fig. 11).

To control the calculations in Fig. 11(3) also shows the phase shift in the absence of a streamer in the discharge gap.

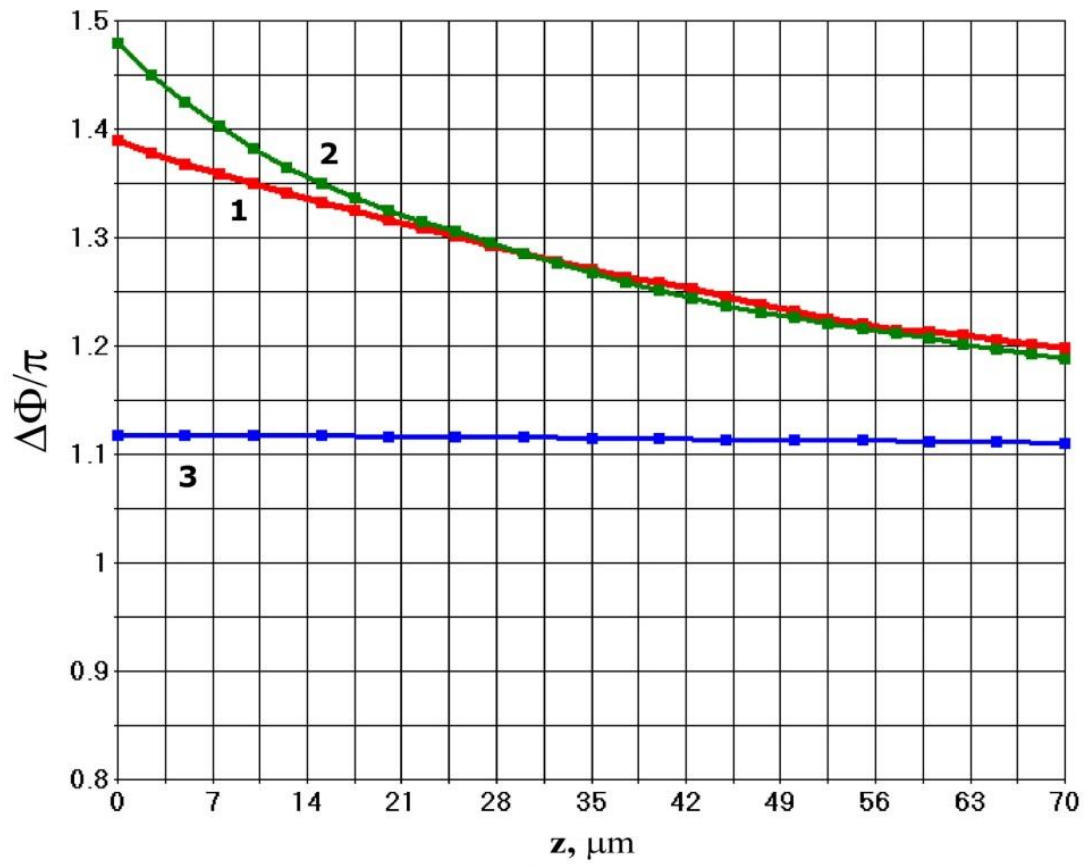

Fig. 11.1 is $\varepsilon=80,2$ is $\varepsilon=\varepsilon(\mathrm{E}), 3$ is without streamer

Thus, we can conclude that the maximum value of the average electric field strength near the streamer head when it propagates from the cathode is observed near the streamer head and does not exceed $E=$ 


\section{$2.2 \mathrm{MV} / \mathrm{cm}$.}

Since the real streamer does not have the correct geometric shape, changes were made to the shape of the streamer cap at the second stage of modeling. Assuming that the streamer's charge is on the wavy surface of the streamer.

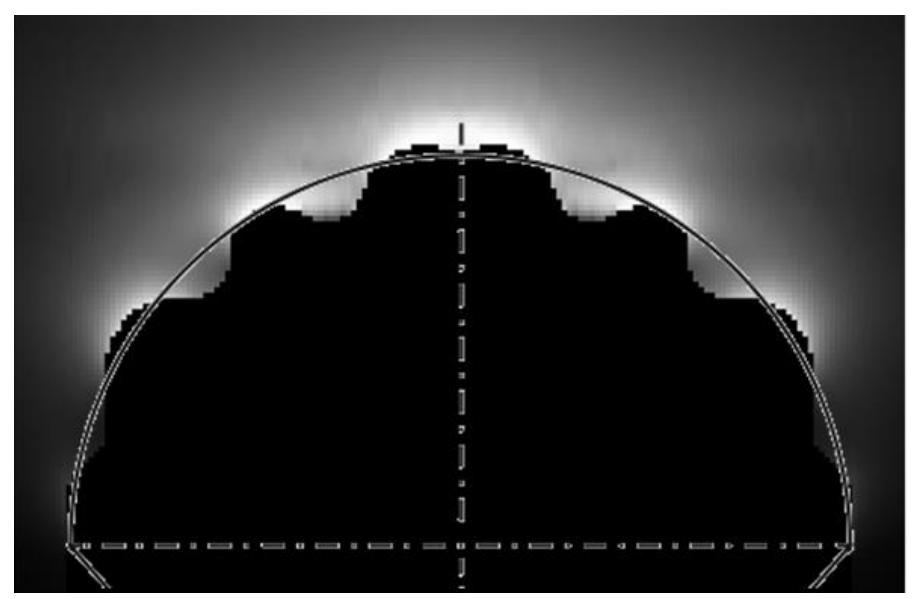

Fig.12. Distribution of $E$ over the surface of the modified streamer model. White color is the maximum $E$. For comparison, the contours of a smooth model are superimposed.

Fig. 13 shows the results of the calculation of $E$, with the modified streamer model taking into account the nonlinearity $\varepsilon$.

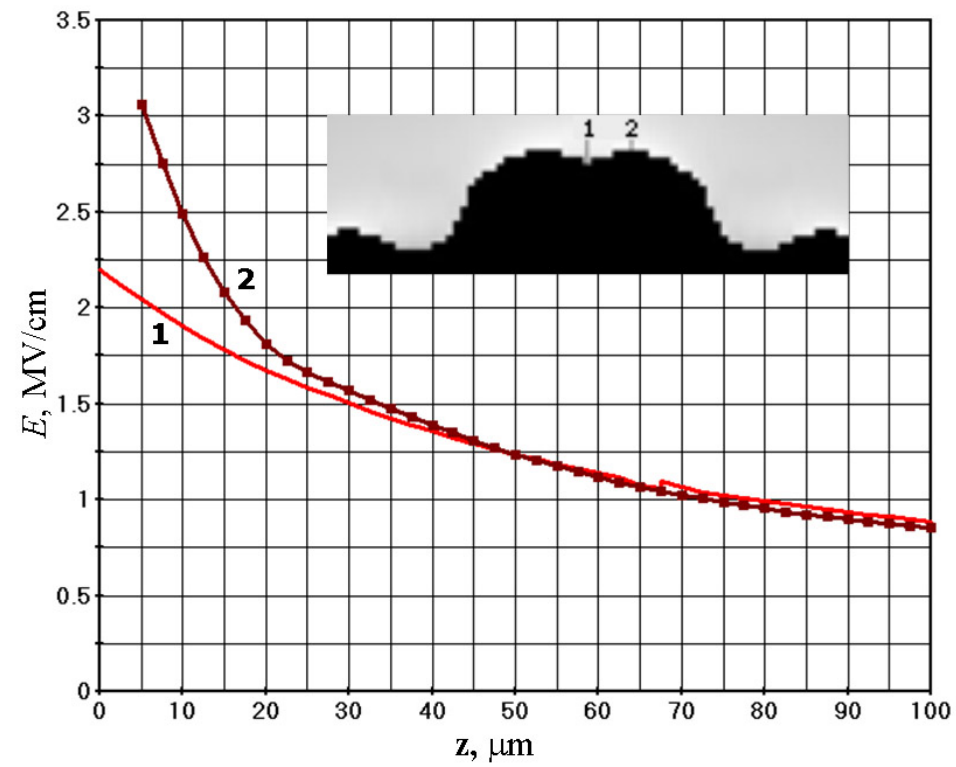

Fig.13. Projection of the vector E onto the $\mathrm{z}$-axis with a modified surface of the streamer model. The distance between points 1 and 2 is $15 \mu \mathrm{m}$.

Comparison of the obtained value $E=3.1 \mathrm{MV} / \mathrm{cm}$ for the modified streamer model with a smooth surface $(E=2.2 \mathrm{MV} / \mathrm{cm})$ showed that the field reaches and even slightly exceeds the threshold value $E_{\mathrm{S}}=3$ $\mathrm{MV} / \mathrm{cm}$, at which possible dipole saturation. However, the area in which this is observed is so small that it has almost no effect on the appearance of the kerrogram (see Fig. 14). 


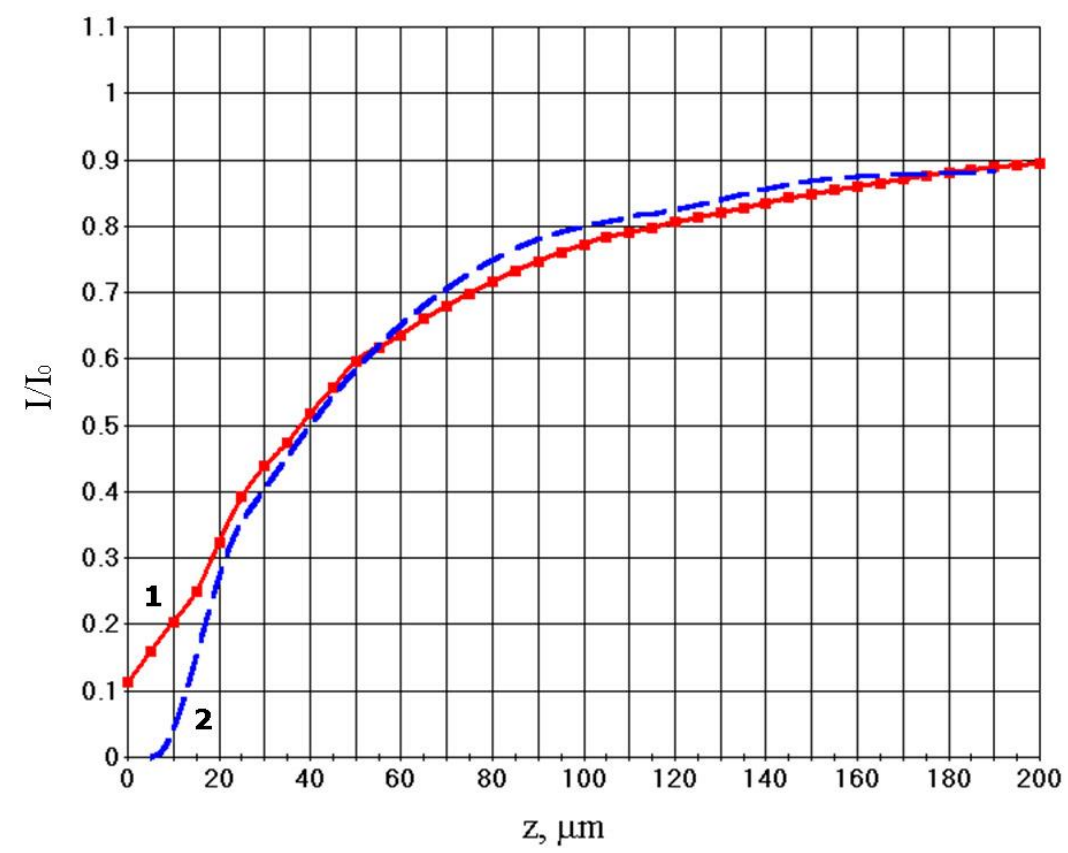

Fig.14. Relative intensities $\mathrm{I} / \mathrm{I}_{0}$ along the $\mathrm{z}$-axis: 1 is smooth model, 2 is modified streamer model.

From the foregoing, we can conclude that the nonlinear dependence of the relative permittivity on the field strength under the conditions under consideration does not lead to any significant change in the result (see Fig. 14). And for this case, $\varepsilon$ can be considered a constant.

In contrast to the experimental kerrogram, where a narrow light region of variable thickness is located between the streamer surface and the black stripe, the black stripe on the calculated kerrogram starts immediately from the surface of the streamer model.

In our opinion, this fact is explained as follows.

As can be seen from Fig. 15 light areas are present only opposite the hollows on the front surface of the streamer.

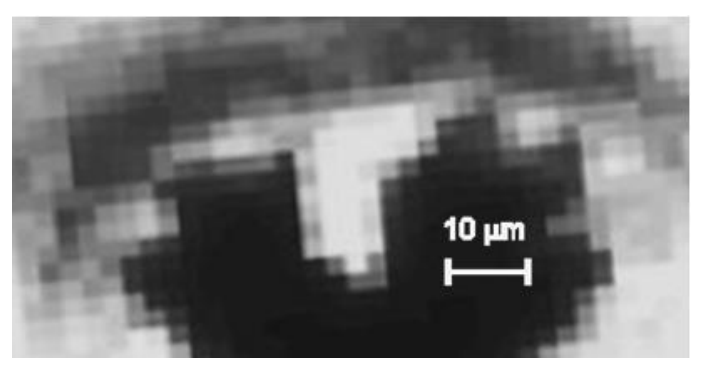

Fig. 15. Area of contact of the front surface of the streamer with the Kerr fringe

In these areas, the electric field is weakened, which is clearly seen in Fig. 13. The phase shift decreases, and the relative intensity of the radiation transmitted through the Kerr cell increases. That is, the field of observation brightens.

Since the dark Kerr fringe closely adjoins only to the protrusions ("prominences") of the streamer, it becomes clear that it is the decrease in the field strength in the hollows of the streamer head that explains the visible variable light gap on the experimental kerrogram.

Consequently, the apparent separation of the dark Kerr fringe from the streamer in the experimental kerrogram is explained by the strong inhomogeneity of the surface of its head. The calculated width of this 
light gap is $\sim 4-6 \mu \mathrm{m}$, which is in good agreement with the experimental kerrogram.

Another explanation is also possible. It is striking that in the experiment there are no sharp changes in the shape of the fringes near the "prominences" on the surface of the streamer. With what it can be connected? In our opinion, the most plausible is the following version based on the EHD mechanism of cathodes streamer development [16]. In front of the streamer, there must be a negative space charge generated by this streamer. The field is strengthened in front of the space charge, and behind it, closer to the streamer body, it is weakened. In addition, the space charge, due to the electrostatic repulsion of ions, "smears" the field and reduces its inhomogeneity. This also leads to "smearing" of the Kerr image and can explain the absence of sharp changes in the fringe shape.

It seems that both of these causes are at work, leading together to an almost regular shape of the dark fringe.

As for the second implicit fringe in Fig. 4a, then it most likely corresponds to a shock wave generated by a fast-moving streamer. The rarefaction zone behind the leading edge of the shock wave does not introduce a noticeable phase shift between the ordinary and extraordinary beams. Therefore, the sufficiently large width of this weak fringe indicates that we actually observe a shadow pattern of the shock wave rarefaction zone and not a Kerr fringe.

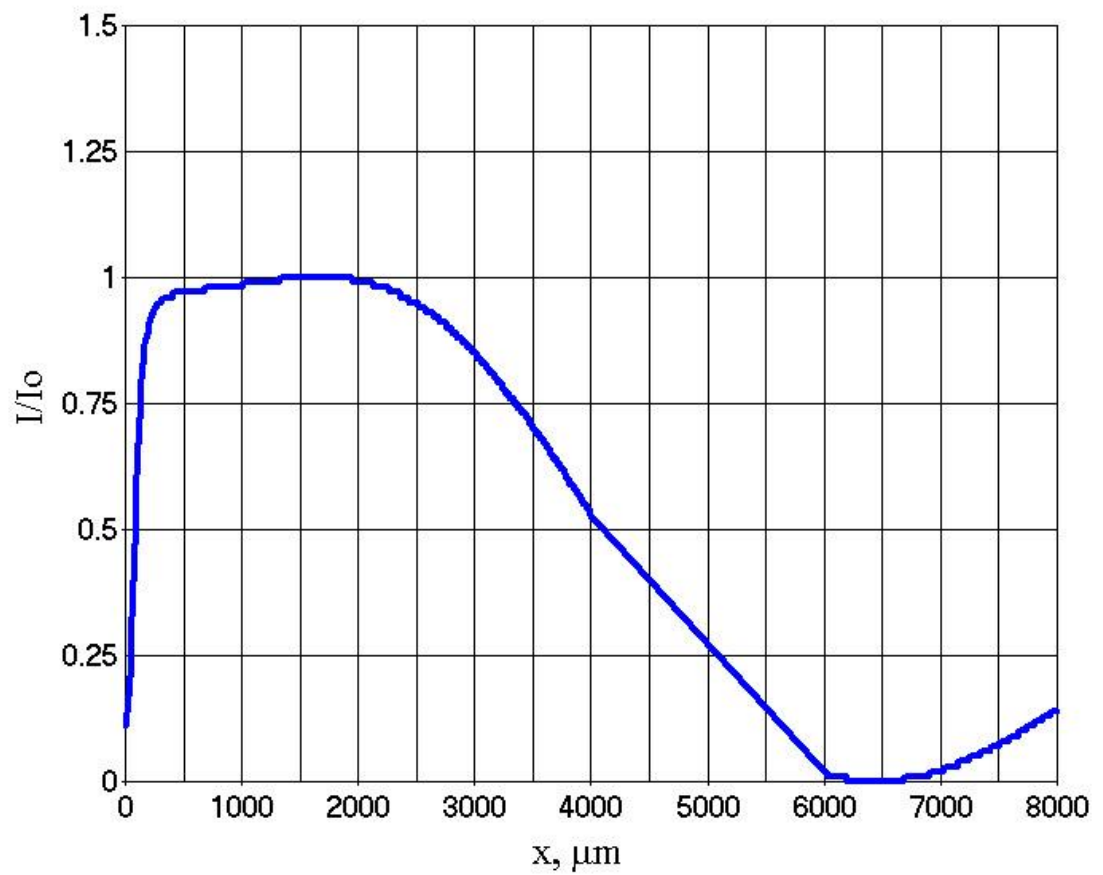

Fig. 16. Distribution of relative intensity $\mathrm{I} / \mathrm{I}_{0}$ and phase shift $\Delta \Phi / \pi$ along the $\mathrm{X}$-axis across the entire observation area. The radius of the probing laser beam $\sim 7-8 \mathrm{~mm}$

But on the periphery of the observation zone (see Fig. 2) on the graph of Fig. 16, a dark Kerr fringe is clearly present, which appears due to a decrease in the field strength and a decrease in the phase shift towards the periphery of the electrode system. The calculated position of this band correlates well with the experimental kerrogram.

\section{CONCLUSIONS}

Thus, modeling the electric field and kerrograms near the cathode streamer made it possible to estimate the field strength in front of its head. This value of the electric field does not exceed $3.1 \mathrm{MV} / \mathrm{cm}$, in contrast to the field strength of the anode streamer [14] determined at 40-50 MV/cm. 
Secondly, we can conclude that the nonlinear dependence of the relative permittivity on the field strength under the conditions under consideration does not lead to any significant change in the result (see Fig. 14), and for this case $\varepsilon$ can be considered a constant.

\section{ACKNOWLEDGMENT}

The work was financially supported by the Ministry of Science and Higher Education of the Russian Federation (Research Laboratory "Modeling and data processing of high technologies," the project code is FSUN-2020-0012).

\section{REFERENCES}

[1] Joshi, R.P., \& Thagard, S.M. (2012). Streamer-Like Electrical Discharges in Water: Part I. Fundamental Mechanisms. Plasma Chemistry and Plasma Processing, 33(1), 1-15. DOI:10.1007/s11090012-9425-5

[2] Baránková, H., \& Bárdos, L. (2010). Atmospheric Pressure Plasma Sources and Processing. Handbook of Deposition Technologies for Films and Coatings, 865-880. DOI:10.1016/b978-0-81552031-3.00017-x

[3] Kolb, J.F., Joshi, R.P., Xiao, S., \& Schoenbach, K.H. (2008). Streamers in water and other dielectric liquids. Journal of Physics D: Applied Physics, 41(23), 234007. DOI:10.1088/0022$3727 / 41 / 23 / 234007$

[4] Bruggeman, P., \& Leys, C. (2009). Non-thermal plasmas in and in contact with liquids. Journal of Physics D: Applied Physics, 42(5), 053001. DOI:10.1088/0022-3727/42/5/053001

[5] Sun, A., Zhuang, J., \& Huo, C. (2016). Formation mechanism of streamer discharges in liquids: a review. High Voltage, 1(2), 74-80. DOI:10.1049/hve.2016.0016

[6] Sacchi, C. A. (1991). Laser-induced electric breakdown in water. Journal of the Optical Society of America B, 8(2), 337. DOI:10.1364/josab.8.000337

[7] Lehtinen, N.G.; Marskar, R. What Determines the Parameters of a Propagating Streamer: A Comparison of Outputs of the Streamer Parameter Model and of Hydrodynamic Simulations. Atmosphere 2021, 12, 1664. https://doi.org/10.3390/ atmos 12121664

[8] Vatamanu, J., \& Bedrov, D. (2015). Capacitive Energy Storage: Current and Future Challenges. The Journal of Physical Chemistry Letters, 6(18), 3594-3609. DOI:10.1021/acs.jpclett.5b01199

[9] Ruma, Lukes, P., Aoki, N., Spetlikova, E., Hosseini, S. H. R., Sakugawa, T., \& Akiyama, H. (2013). Effects of pulse frequency of input power on the physical and chemical properties of pulsed streamer discharge plasmas in water. Journal of Physics D: Applied Physics, 46(12), 125202. DOI:10.1088/0022-3727/46/12/125202

[10] Sarkisov G.S., and Woodworth J.R. Observation of electric field enhancement in a water streamer using Kerr effect. Journal of Applied Physics 99, 083304 (2006); DOI:10.1063/1.2189215

[11] Korobeynikov, S.M., \& Melekhov, A.V. (2014). Estimations of the electric field strength of nonelectrode streamers in water. High Temperature, 52(1), 129-131. DOI:10.1134/s0018151x14010118

[12] Dobrynin, D., Seepersad, Y., Pekker, M., Shneider, M., Friedman, G., \& Fridman, A. (2013). Nonequilibrium nanosecond-pulsed plasma generation in the liquid phase (water, PDMS) without bubbles: fast imaging, spectroscopy and leader-type model. Journal of Physics D: Applied Physics, 46(10), 105201. DOI:10.1088/0022-3727/46/10/105201 
[13] Starikovskiy, A., Yang, Y., Cho, Y. I., \& Fridman, A. (2011). Non-equilibrium plasma in liquid water: dynamics of generation and quenching. Plasma Sources Science and Technology, 20(2), 024003. DOI:10.1088/0963-0252/20/2/024003

[14] Yassinskiy V.B., Kuznetsova Yu.A., Korobeynikov S.M., and Vagin D.V. Simulation of electrooptical measurements of prebreakdown electric fields in water. Part 1. Electric field near anode streamer. - https://doi.org/10.36227/techrxiv.19185227.v1

[15] Wagenaars, E., Bowden, M. D., \& Kroesen, G. M. W. (2007). Measurements of Electric-Field Strengths in Ionization Fronts during Breakdown. Physical Review Letters, 98(7). DOI:10.1103/physrevlett.98.075002

[16] Korobeynikov, S.M., Melekhov, A.V., Posukh, V.G., Ponomarenko, A.G., Boyari, E., \& Antonov, V.M. (2009). Optical study of prebreakdown cathode processes in deionized water. IEEE Transactions on Dielectrics and Electrical Insulation, 16(2), 504-508. DOI:10.1109/tdei.2009.4815185

[17] Korobeynikov S.M., Kuznetsova Yu.A., Yassinskiy V.B. Simulation of electrooptical experiments in liquids. Journal of Electrostatics. Volume 106, July 2020, 103452 https://doi.org/10.1016/j.elstat.2020.103452

[18] Booth F. The Dielectric Constant of Water and the Saturation Effect. J. Chem. Phys. 19. 391 (1951); https://doi.org/10.1063/1.1748233 